\title{
Recurrent Ulceration after Vagotomy and Drainage with Electrical Stimulation Test, 1957-69
}

\author{
H. BURGE,* M.B.E., M.B., F.R.C.S.; R. D. STEDEFORD, † PH.D., B.M., F.R.C.S.; D. HOLLANDERS, $\ddagger$ M.B., B.S., M.SC.
}

\begin{abstract}
Cummary: Between 1957 and 1969700 patients with duodenal ulceration were treated by selective vagotomy and simple drainage using the electrical stimulation test to achieve complete nerve section. Ten of these patients have been re-admitted to the hospital with further ulceration, one with a lesser curve gastric ulcer and nine with recurrent duodenal disease. The first patient had gastric retention and has apparently been cured by gastrojejunostomy to improve antral drainage. The remaining nine cases were found to have incomplete nerve section, and, of these, seven have been treated and apparently cured by completion of the incomplete vagotomy, again using the electrical stimulation test.
\end{abstract}

\section{Introduction}

The electrical stimulation test for completeness of gastric vagotomy (Burge and Vane, 1958) was first used at the West London Hospital in 1957. Since then it has been used in every case, some 700 in all. This paper records the recurrent cases in this series which have come under our care for investigation and treatment.

Several factors bedevilled this test in its early years. Above all we did not then properly understand the problem of anticholinergic drugs given either before or during the operation. Many preparations used therapeutically in the treatment of peptic ulceration contain an anticholinergic agent, which, if taken regularly during the days or weeks preceding operation, will diminish or abolish the gastric response to stimulation.

The anaesthesia for almost all these patients was carried out by two anaesthetists, one of whom used halothane (Fluothane) and the other did not. Only repeated failure of the preliminary test to function properly on Fridays led us to the discovery that halothane, even in small concentrations, would abolish or severely diminish the response to vagal stimulation. There was, too, the problem of incorrect premedication which arose from time to time in spite of every care. The preliminary stimulation test is important. If a satisfactory response is not obtained, then an anticholinergic drug must have been given and the final test is invalid.

The vagotomy in all these patients has been selective. In some either anterior or posterior selective nerve section was used, but most were treated by the bilateral selective method. The prepyloric gastric branches arising from the anterior vagus through the hepatic plexus and its pyloric branches were not divided.

Ten patients with recurrent ulceration have come under our care for investigation and treatment.

\section{Case Reports}

Case 1.-This patient presented with recurrent duodenal ulceration two months after vagotomy and pyloroplasty. The operation was performed by a surgeon not experienced in vagotomy. 'Difficulty arose in an attempt to preserve an ectopic left hepatic artery. The descending branches of the artery were left undivided. An insulin test 10 days after operation was positive (Fig. 1 a). A further insulin test a year later was also positive (Fig. 1 b). At a second operation the electrical test was applied initially with the

\footnotetext{
* Surgeon, West London Hospital, London W.6.

+ Resident Assistant Surgeon, West London Hospital, London W.6.

† House-surgeon, West London Hospital, London W.6.
}

posterior vagus outside the electrode. Incomplete anterior vagotomy was found (Fig. 2). The incomplete anterior nerve section was completed and then proved by test (Fig. 3). The posterior nerve was then included in the electrode and incomplete posterior section shown (Fig. 4). This was completed by division of the descending branch of the left gastric artery. Finally, complete gastric vagotomy was proved (Fig. 5). The insulin test became negative (Fig. $1 \mathrm{c}$ ) and the patient remained well. Cause of recurrent ulceration: incomplete vagotomy.

Case 2.-Unfortunately there were no records of the responses to stimulation, but the stimulation test was used and vagotomy thought to be complete. Ten days after operation an insulin test was negative (Fig. $1 \mathrm{~d}$ ). A further test 15 months later was equivocal (Fig. 1 e). Three months after this two tests were positive (Fig. $1 \mathrm{f}$ and g). At a second operation for recurrent duodenal ulcer two years after the first the stimulation test was used. The preliminary test gave a pressure rise of $1.5 \mathrm{~cm}$. $\mathrm{H}_{2} \mathrm{O}$. The incomplete vagotomy was completed, after which the stimulation test showed no rise of pressure. A further insulin test ten days later was negative (Fig. $1 \mathrm{~h}$ ), but when this test was repeated five and six months postoperatively a late positive result was obtained on both occasions. Cause of recurrent ulceration: incomplete vagotomy.

Case 3.-Selective vagotomy and gastrojejunostomy was performed for duodenal ulceration in 1960. The anaesthetic was administered by the anaesthetist who routinely used halothane. In 1962 a second operation was performed for recurrent duodenal ulcer. The preliminary response to the stimulation was $3 \mathrm{~cm}$. $\mathrm{H}_{2} \mathrm{O}$. The vagotomy was complete after four small nerve trunks were found and divided. These nerves were examined histologically. The gastrojejunostomy was taken down and replaced by a pyloroplasty. For six years he remained well until admitted with melaena. He had been taking aspirin. An insulin test at this time was perhaps positive but difficult to interpret (Fig. 1 i). The patient remains well. Cause of recurrent ulceration: incomplete vagotomy.

Case 4.-Vagotomy and pyloroplasty was performed in 1966. The descending branches of the left gastric artery were not divided, an attempt being made to divide the gastric division of the posterior vagus, leaving the coeliac division intact without dividing any of the descending branches of the gastric artery. At the end of the operation a rise of pressure of $6 \mathrm{~mm} . \mathrm{H}_{2} \mathrm{O}$ was recorded on the stimulation test. The nerve causing the rise could not be found. Twenty months later recurrent duodenal ulceration was proved on $x$-ray examination. The insulin test in January 1969 was positive (Fig. 1 j). At the second operation incomplete nerve section was shown by the stimulation test. The rise of pressure at the preliminary test was $3 \mathrm{~cm}$. $\mathrm{H}_{2} \mathrm{O}$. The incomplete section was completed and proved on test. The insulin test 12 days later was negative (Fig. $1 \mathrm{k}$ ). This test was repeated five weeks later and was also negative (Fig. 1 1). Cause of recurrent ulceration: incomplete vagotomy.

Case 5.- Selective vagotomy and pyloroplasty together with a Nissen fundoplication was done for hiatus hernia and duodenal ulceration in May 1966. The preliminary stimulation test gave no response, apparently because the patient had been given chlorpromazine (Largactil) until the day of the operation. Five months later a recurrent duodenal ulcer was evident on $x$-ray examination, and the insulin test was positive both at 15 months and at two years (Fig. $1 \mathrm{~m}$ and $\mathrm{n}$ ). Incomplete vagotomy was proved at a second operation by the stimulation test. The vagotomy was completed and the insulin test was negative four months later (Fig. 1 o). Cause of recurrent ulceration: incomplete vagotomy.

Case 6.-Anterior selective vagotomy and Finney pyloroplasty was carried out in June 1962. Symptoms returned in June 1965. The patient was vomiting food. Barium studies showed a lesser curve gastric ulcer and gastric retention (Fig. $6 \mathrm{a}$ and b). The 


\section{$\Delta=$ INSULIN}

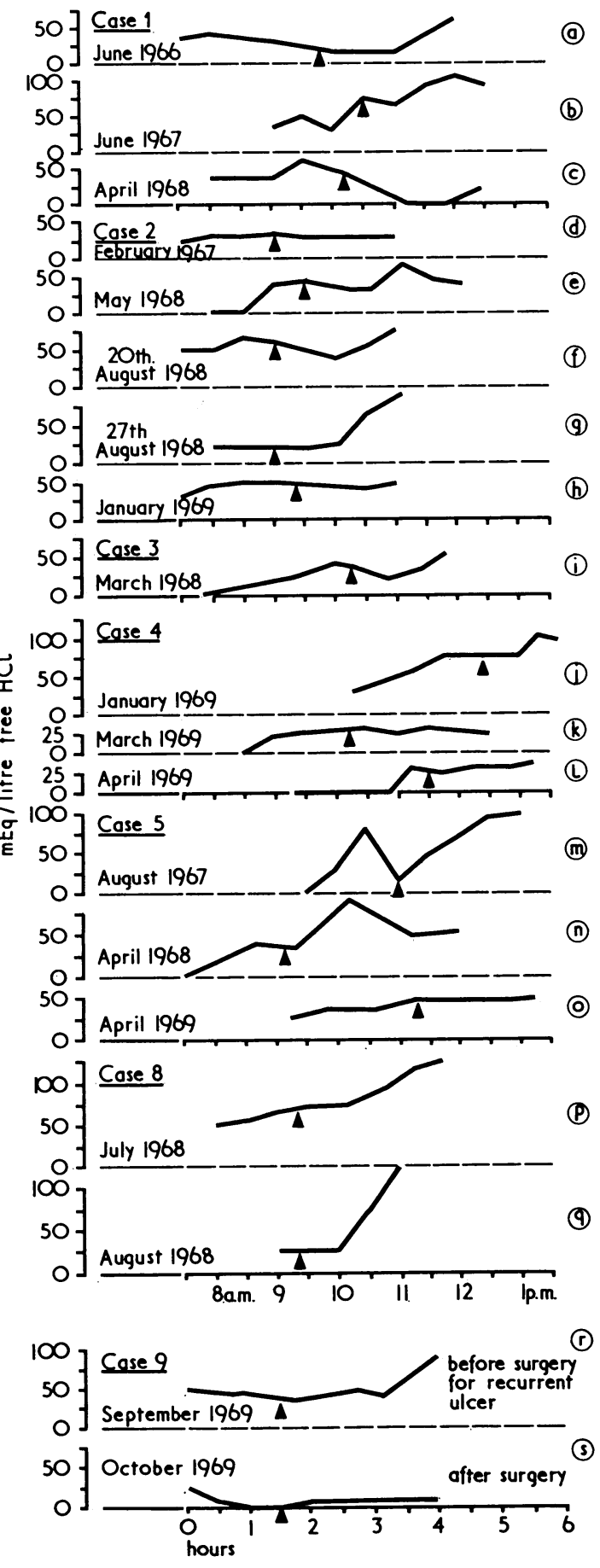

Fig. 1.-Insulin test studies.

Finney pyloroplasty was short-circuited by a gastrojejunostomy. The gastric ulcer remained healed, but the patient suffered from persistent episodic diarrhoea, which was not present before the gastrojejunostomy was made. Cause of lesser curve ulcer: gastric retention following vagotomy and Finney pyloroplasty.

Case 7.-Selective vagotomy and gastrojejunostomy was performed for stenosing duodenal ulceration in November 1958. The stimulation test at the operation showed a complete vagotomy. The patient remained well for two years. Symptoms returned and he was found to have a large gastrojejunal ulcer and antral retention (Fig. $7 \mathrm{a}$ and b). He refused further operation. In December 1960 he was found to be quite well and a barium meal showed no evidence of the ulcer. Eighteen months later no definite ulcer could be found on barium-meal examination. This patient remains well Cause of transient gastrojejunal ulcer: transient antral retention.
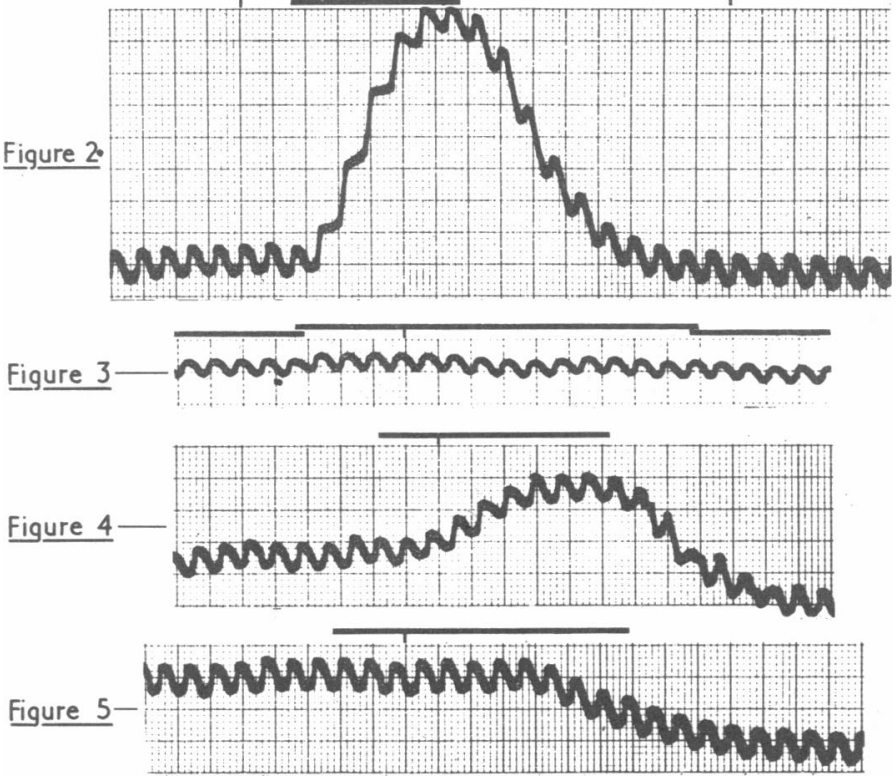

Fig. 2.-Incomplete anterior vagotomy. FIG. 3.-Complete anterior vagotomy. FIG. 4.-Incomplete posterior vagotomy. FIG. 5.-Complete posterior vagotomy.

Case 8.-Bilateral selective vagotomy and pyloroplasty was done in a 16-year-old patient with advanced duodenal stenosis. When the patient was seen five years later with recurrent duodenal ulceration the insulin test was positive (Fig. 1 p). At a second operation it was found that the left gastric artery had not been divided. Nevertheless, preliminary stimulation test at the operation was negative, as was the final test. One month later the insulin test was again positive (Fig. 1 q). At a further operation an anterior duodenal ulcer was found. It was not possible to free the oesophagus safely for yet another electrical stimulation test, so antrectomy was performed. Cause of recurrent ulceration: incomplete vagotomy.

Case 9.-Bilateral selective vagotomy and pyloromyotomy was performed for duodenal ulcer and lesser curve gastric ulcer in December 1967. Vagotomy was thought complete according to the electrical stimulation test, though a note recorded that there was some difficulty with the test because of fibrillation of the electrode due to current leakage to the crura of the diaphragm. A year later symptoms recurred and a duodenal ulcer was shown on $x$-ray examination. An insulin test in September 1969 showed a late positive response (Fig. $1 \mathrm{r}$ ). This was at once repeated, with the same result. At subsequent operation a recurrent duodenal ulcer was found together with a large intact anterior trunk which produced a considerable response on stimulation. When the trunk was divided nerve section was shown complete (Fig. 8 a, b). An insulin test 10 days later was negative (Fig. $1 \mathrm{~s}$ ). This rather large and oedematous nerve seemed too big to have been overlooked at the first operation, and the possibility of nerve regeneration should perhaps be considered. Cause of recurrent ulceration: incomplete vagotomy.

Case 10.-At operation complete vagotomy could not be achieved. The electrical stimulation test showed a persistent rise of $3 \mathrm{~mm}$. of water pressure. After many attempts to find any small intact nerves, the search was abandoned. After this the small response was abolished by atropine. Within a few months this patient had recurrent symptoms and melaena. The insulin test was strongly positive. He is at present under observation. Cause of recurrent ulceration: incomplete vagotomy.

\section{Discussion}

Of some 700 patients with peptic ulceration treated since 1957 by selective vagotomy and drainage, using the electrical stimulation test, 10 have returned to the West London Hospital with recurrent ulceration for investigation and treatment. One had a lesser curve gastric ulcer with gastric retention proximal to a Finney pyloroplasty. This patient has remained well since the gastric antrum was drained by gastrojejunostomy in 1965. This simple drainage operation, however, has 

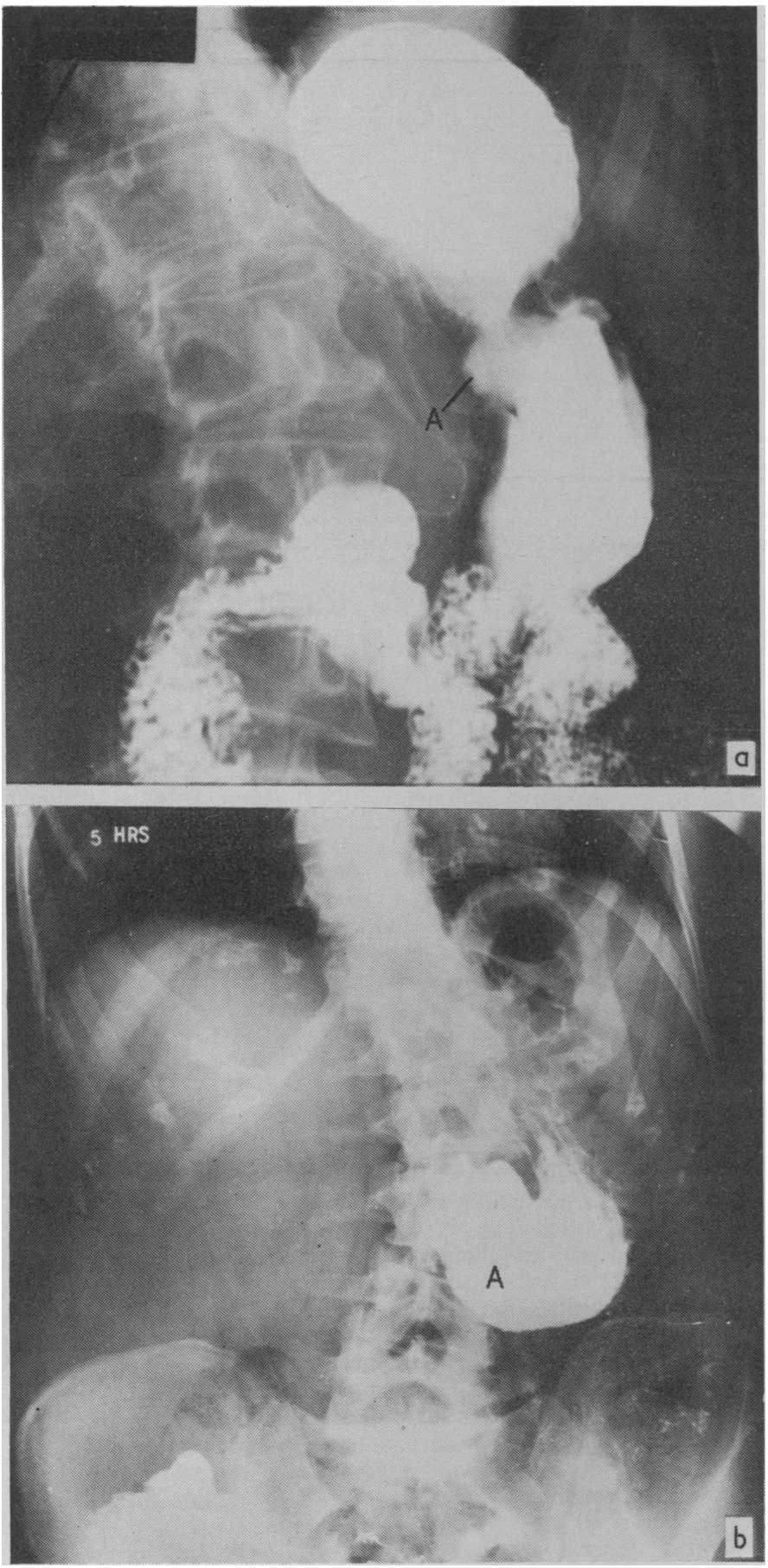

FIG. 6.-(a) Lesser curve gastric ulcer (A) after selective vagotomy and and Finney pyloroplasty. (b) Gastric retention.

left her with persistent episodic diarmoea. In another patient gastric antral retention between the gastrojejunostomy and a stenosed duodenum gave rise to a large gastrojejunal ulcer. This ulcer healed spontaneously. He has remained well for eight years. The remaining eight patients developed recurrent duodenal ulceration. Of these, six continued to remain well after completion of the incomplete vagotomy. In the seventh case, with an obviously positive insulin test, antrectomy was ultimately performed. The eighth is under observation.

Incomplete vagal section was due in two cases to anticholinergic drugs. In one chlorpromazine was given daily before the operation, and in the other, it seems, halothane was used as an anaesthetic agent. In three of the other four patients with incomplete nerve section an attempt was made at the first operation to divide the gastric division of the posterior vagus without dividing the descending branch or branches of the left gastric artery (Fig. 9). This simple tech-

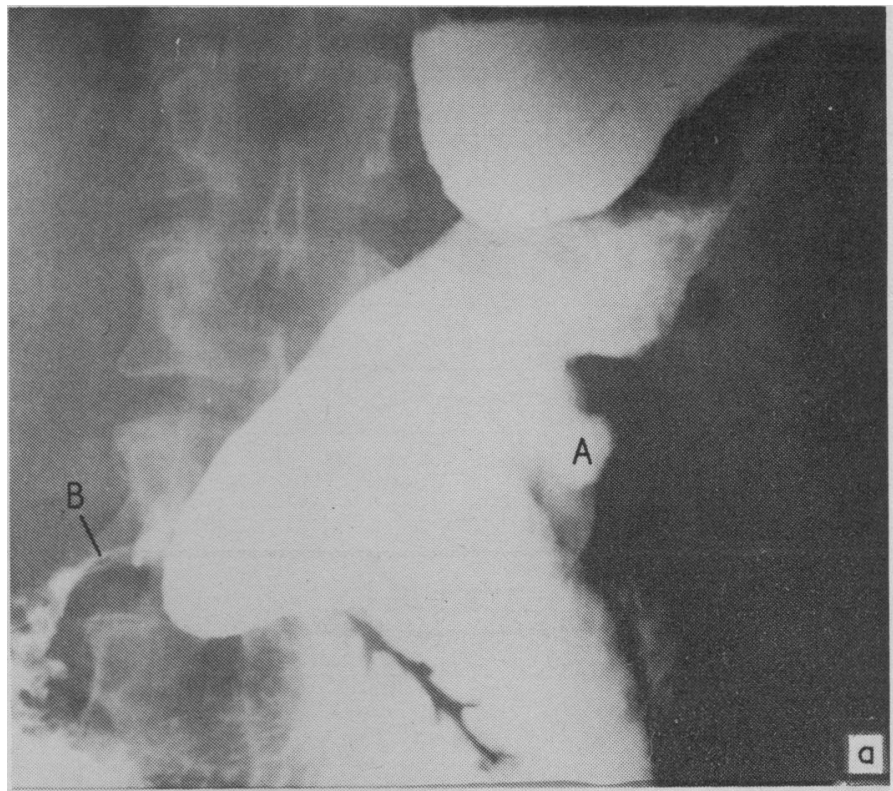
simple drainage if nerve section to the stomach is complete and

FIG. 7.- (a) Gastrojejuned ulcer after vagotomy and gastrojejunostomy. $A=$ Gastrojejunal ulcer. $B=$ Stenosed duodenum. (b) $A=$ Antral retention.

nique should never be used, for, as Jackson (1949) showed, gastric branches may arise from the coeliac division itself (Fig. 10). When the operation is done in this way the "final" electrical test is not satisfactory. The posterior trunk cannot properly be stimulated because of the mass of tissue which surrounds it and the ascending artery and which must be included in the elecrode.

Now that we are able to study these cases of recurrent ulceration it is clear that a very small retained vagal trunk, giving rise to only a few millimetres of water pressure with the stimulation test, may be responsible for recurrent ulceration. We believe from our studies that the concept of adding antrectomy or mucosal antrectomy is based on an incorrect hypothesis and there is no need to do anything more than vagotomy and the antrum properly drained. Indeed recent studies suggest that in chosen cases even the drainage operation may be omitted (Burge et al., 1969). In order to achieve complete gastric 


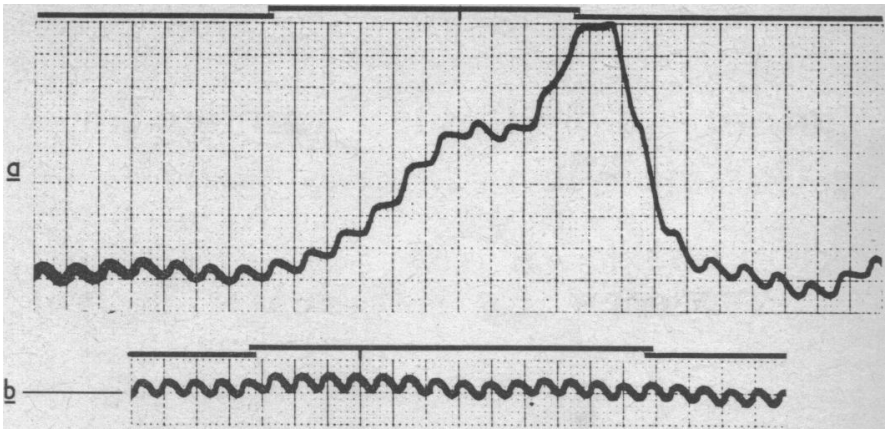

FIG. 8.-(a) Incomplete nerve section from large intact anterior trunk. (b) Complete nerve section.
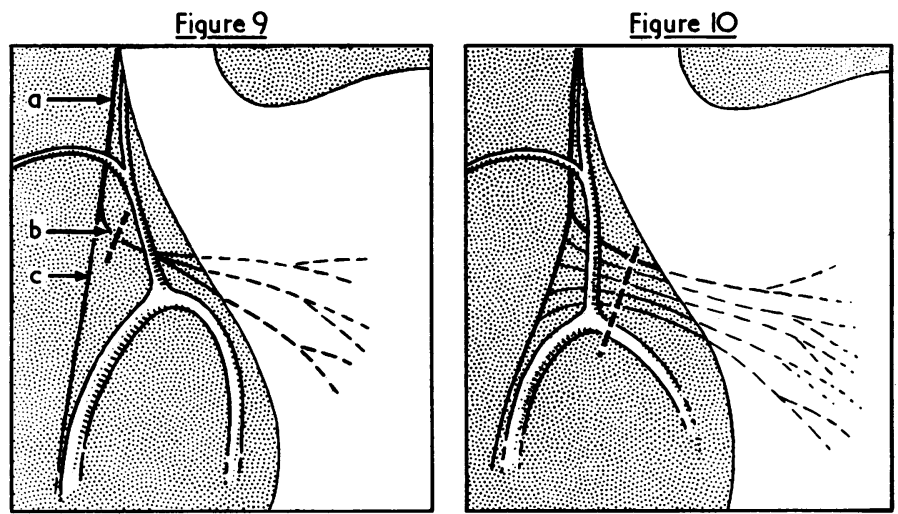

FIG. 9.-Division of posterior vagus, leaving arteries completely intact. $a=$ Posterior vagus nerve. $b=$ Gastric division of posterior nerve. $c=$ Coeliac division of posterior nerve.

FIG. 10.-Gastric branches arising from coeliac division of posterior vagus (after Jackson, 1949).

vagotomy a test for completeness of nerve section at the time of operation is essential. The instrument now recommended for the electrical stimulation test is the Burge electronic Vagotometer manufactured by Furness Controls Limited of Bexhill, England. The prototype of this is shown in Fig. 11. Working to a sensitivity of a 3-mm. rise in water pressure, we would at present leave $80 \%$ of patients with an incomplete vagotomy had the test not been used.

An assessment of the leucomethylene blue test (Lee, 1969) has shown it to be unsatisfactory (Burge, 1970; Cooke et al., 1970; Jensen et al., 1970). In making use of the insulin test to assess completeness of vagotomy it became apparent that gastric acid levels can vary spontaneously to a marked degree before insulin was given. The variations were large enough in many cases-see Fig. 1 b, e, $f, m-$ to be taken as evidence of incomplete vagotomy had they occurred during the period of hypoglycaemia.

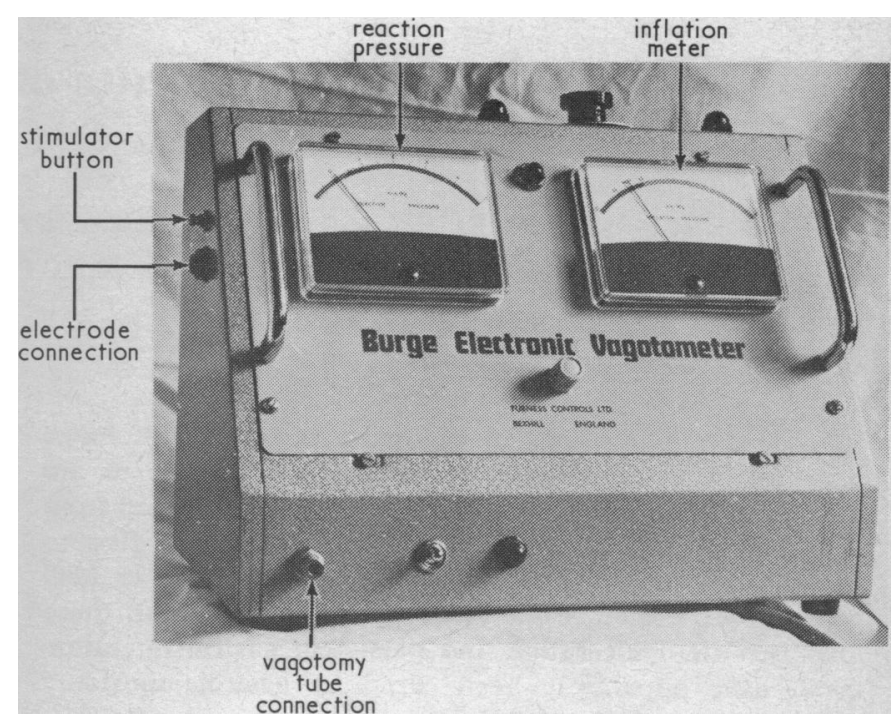

FIG. 11.-Prototype of the Burge electronic Vagotometer.

Consequently it is emphasized that a period of time is required before giving insulin (two hours in the present work), during which the levels of gastric acidity can be followed. Reliance on the interpretation of post-insulin acidities should be placed only in the light of the pre-insulin production: that is, greatest reliance can be attached to those results in which pre-insulin acid levels were either constant or falling slowly-for example, Fig. 1 a, d, g, o.

Finally, this long-term study confirms Dragstedt's very early opinion that recurrent duodenal ulcer after vagotomy is due always to incomplete nerve section. It can therefore be prevented by the correct use of the electrical stimulation test. The only gastric ulcer occurring after vagotomy for duodenal ulcer in this series also supports Dragstedt's hypothesis that this condition is due to gastric retention. The ulcer was cured by overcoming gastric retention by gastrojejunostomy. If these facts are true, then no case can be made for preoperative acid studies in planning the surgical treatment of duodenal disease.

\section{REFERENCES}

Burge, H. (1970). Lancet, 1, 950

Burge, H., MacLean, C., Stedeford, R., Pinn, G., and Hollanders, D. (1969). British Medical fournal, 3, 690.

Burge, H., and Vane, J. R. (1958). British Medical fournal, 1, 615.

Cooke, W. M., Talbot, I. C., Welbourn, R. B., and Cox, A. G. (1970). Lancet, 1, 864.

Lancet, 1, 864.
Jackson, R. G. (1949). Anatomical Record, 103, 1.

Jackson, R. G. (1949). Anatomical Record, 103, 1.
Jensen, H. E., Nielsen, J., Poll, P., and Amdrup, E. (1970). 4th World Congress of Gastroenterology. Copenhagen. 12-18 July. Advance Abstracts, p. 438

Lee, M. (1969). British fournal of Surgery, 56, 10. 